\title{
Tracios en Hispania ${ }^{1}$
}

\section{Thracians in Hispania}

\section{JOSÉ ORTIZ CÓRDOBA Universidad de Granada joseortiz@ugr.es}

\begin{abstract}
Resumen: Este trabajo tiene como objetivo principal la recopilación y estudio de la documentación epigráfica relacionada con la presencia de inmigrantes de origen tracio en la península ibérica. Pretendemos ofrecer un catálogo epigráfico actualizado y una síntesis de esta emigración. Entre otros elementos se estudiarán las causas que motivaron los desplazamientos, la onomástica de los inmigrantes detectados y la actividad que desarrollaron en Hispania.
\end{abstract}

Palabras clave: emigración, movilidad, Tracia, Hispania, epigrafía.

Abstract: The main objective of this article is to compile and study the epigraphic documentation related to the presence of Thracian immigrants origin in the Iberian Peninsula. We aim to offer an updated epigraphic catalogue and a synthesis of this emigration. Among other elements, the causes that motivated the displacements, the onomastics of the detected immigrants and the activity that they developed in Hispania will be studied.

Keywords: emigration, mobility, Thrace, Hispania, epigraphy.

\footnotetext{
${ }^{1}$ Ayuda puente doctores Plan Propio. Departamento de Historia Antigua, Universidad de Granada. Este trabajo se enmarca en las líneas de estudio del grupo de investigación HUM-215 dirigido por el Prof. Dr. C. González Román y deriva de la tesis doctoral Las colonias romanas de Hispania y los movimientos de población (siglos I-II d.C.), defendida en la Universidad de Granada en marzo de 2019. Asimismo, forma parte del proyecto de investigación Veterani et milites en las colonias romanas de Hispania, otorgado a quien suscribe estas líneas por el Plan Propio de Investigación de la Universidad de Granada en el marco del Programa de Proyectos de Investigación para Jóvenes Investigadores.
} 


\section{Introducción}

Tracia conformaba una amplia región situada en el extremo sureste de la península de los Balcanes. Sus habitantes, famosos por su habilidad para cabalgar, constituían un complejo conglomerado de tribus organizadas de forma autónoma. Por su estratégica situación geográfica, la región se convirtió desde muy pronto en una zona clave para controlar el paso entre Europa y Asia a través del Bósforo. Durante el siglo II a. C., Roma, tras la anexión de Macedonia, procedió a extender su influencia sobre la zona costera de Tracia con el objetivo de garantizarse un corredor marítimo que le permitiera enlazar con la provincia de Asia. Los contactos con el mundo tracio se intensificaron durante el siglo I a. C., momento en que Roma se aseguró un mejor control de las tierras del bajo Danubio mediante la anexión de la vecina región de Moesia. Durante ese periodo Tracia fue reducida a la categoría de reino cliente, un paso previo a su definitiva conversión en provincia romana durante el gobierno del emperador Claudio (46 d. C.) $)^{2}$.

La peculiar posición geográfica de este territorio condicionó su evolución histórica, marcada por su carácter de tierra de frontera. Las relaciones entre Tracia y la península ibérica no fueron tan estrechas como las mantenidas con otras regiones geográfica y culturalmente más próximas, como el norte de África, la Galia o la propia Italia. No obstante, el estudio de la documentación epigráfica permite constatar la existencia de ciertos contactos entre ambos territorios. Estos presentan un carácter unidireccional, ya que la presencia de individuos de origen tracio en Hispania no fue replicada con la llegada de hispanos a Tracia. Se trata, en cualquier caso, de una movilidad de carácter limitado en la que el ejército tuvo un papel destacado y que cronológicamente remite a los siglos I y III d. C.

La llegada a la península ibérica de gentes procedentes de otros territorios del Imperio ha tenido una notable proyección en la historiografía ${ }^{3}$. Semejante proceso histórico debe ser analizado en el contexto general de los movimientos de población originados tras la conquista romana de Hispania. Este hecho conllevó el establecimiento de un notable flujo inmigratorio compuesto principalmente por itálicos, cuya presencia favoreció la romanización de los territorios ocupados ${ }^{4}$. En los años siguientes la llegada de población itálica a Hispania se

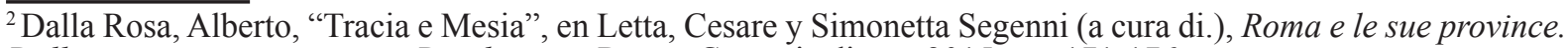
Dalla prima guerra punica a Diocleziano, Roma, Carocci editore, 2015, pp. 171-176.

${ }^{3}$ Albertini, Eugène, "Les étrangers residant en Espagne á l’epoque romaine”, en Mélanges Cagnat, Paris, 1912, pp. 297-318; Balil, Alberto, "La economía y los habitantes no hispánicos del levante español durante el Imperio romano", en Archivo de Prehistoria Levantina, 5 (1954), pp. 251-273; García y Bellido, Antonio, "El elemento forastero en Hispania romana", en Boletín de la Real Academia de la Historia, 144 (1959), pp. 119-154; Haley, Evan W., Foreigners in Roman Imperial Spain: investigations of geographical mobility in the spanish provinces of the Roman Empire (30 B.C. - A.D. 284), Columbia University, 1986, pp. 175-237; Haley, Evan W., Migration and economy in Roman Imperial Spain, Barcelona, Universidad de Barcelona, 1991, pp. 27-52.

${ }^{4}$ Marín Díaz, Ma . Amalia, "La emigración itálica a Hispania en el siglo II a. C.", en Studia Historica. Historia Antigua, 4-5 (1986-1987), pp. 53-63; Marín Díaz, Mª Amalia, Emigración, colonización y municipalización en la Hispania republicana, Granada, Universidad de Granada, 1988; Barreda Pascual, Adela, Gentes itálicas en
} 
incrementó, alcanzando su máximo volumen durante la colonización cesariana y augustea ${ }^{5}$. El final de las guerras civiles que marcaron la última etapa de la República favoreció el desarrollo de la movilidad geográfica, que alcanzó un notable grado de complejidad y una enorme diversidad durante los siglos del Principado. Gracias a la epigrafía conocemos la llegada a Hispania de numerosos inmigrantes, entre los que destacaron por su número itálicos ${ }^{6}$, galos ${ }^{7} \mathrm{y}$ africanos ${ }^{8}$. A ellos debemos sumar otros grupos de población procedentes de regiones más lejanas como Grecia, el este mediterráneo y las zonas fronterizas del Rhin y el Danubio9.

El estudio de estos colectivos ha tenido una proyección desigual. Algunos de ellos, como sucede con los itálicos, los africanos o los orientales, han recibido una notable atención por parte de los investigadores, mientras que otros han pasado prácticamente desapercibidos como consecuencia, podemos imaginar, de su escaso volumen. Entre estos últimos se encuentra la inmigración de origen tracio. Su presencia en el ámbito historiográfico se limita a las breves consideraciones realizadas por A. García y Bellido y E. W. Haley en sus trabajos sobre la emigración en la península ibérica ${ }^{10}$. Carecemos, por tanto, de un estudio específico sobre la movilidad relacionada con este territorio en el que se recoja un listado actualizado de la documentación epigráfica y de las referencias bibliográficas relacionadas con la misma.

Hispania Citerior (218-14 d.C.). Los casos de Tarraco, Carthago Nova y Valentia, tesis doctoral, Universidad de Barcelona, 1998; Le Roux, Patrick, "L'emigration italique en Citérieure et Lusitanie jusqu'à la mort de Néron", en Beltrán Lloris, Francisco (ed.), Roma y el nacimiento de la cultura epigráfica en occidente, Zaragoza, Institución Fernando el Católico, 1995, pp. 85-95; Navarro Caballero, Milagros, "Notas sobre algunos gentilicios romanos de Lusitania: una propuesta metodológica acerca de la emigración itálica", en Gorges, Jean-Gérard y Trinidad Nogales Basarrate (eds.), Sociedad y cultura en Lusitania romana. IV Mesa Redonda Internacional, Mérida, Junta de Extremadura, 2000, pp. 281-297; González Román, Cristóbal, "Romanos e itálicos en la Hispania republicana", en Pons Pujol, Lluís (ed.), Hispania et Gallia: dos provincias del Occidente romano, Barcelona, Universidad de Barcelona, 2010, pp. 13-32.

${ }^{5}$ Para un estado de la cuestión sobre la colonización romana en Hispania ver Ortiz Córdoba, José, Las colonias romanas de Hispania y los movimientos de población (Siglos I-II d.C.), tesis doctoral, Universidad de Granada, 2019, p. 69-531.

${ }^{6}$ Ver nota 4.

${ }^{7}$ García y Bellido, Antonio, "El elemento forastero... op. cit., pp. 129-131; Haley, Evan W., Foreigners in... op. cit., pp. 146-152; Haley, Evan W., Migration and... op. cit., pp. 33-37; Beltrán Lloris, Francisco, "Galos en Hispania", en Acta Archaeologica Academiae Scientiarum Hungaricae, vol. 57, 1-3 (2006), pp. 183-200, https:// doi.org/10.1556/AArch.57.2006.1-3.13; Ortiz Córdoba, José, "De Gallia a Hispania. La inmigración gala en la península ibérica a través de las evidencias epigráficas", en Hispania Antiqua, 43 (2019), pp. 155-201, https://doi. org/10.24197/ha.XLIII.2019.155-201

${ }^{8}$ García y Bellido, Antonio, "El elemento forastero... op. cit., pp. 144-150; Haley, Evan W., Foreigners in... op. cit., pp. 162-174; Haley, Evan W., Migration and... op. cit., pp. 44-51; Gozalbes Cravioto, Enrique, "Documentos epigráficos acerca de las relaciones entre Hispania y Mauretania Tingitana", en Akerraz, Aomar et al. (eds.), L'Africa romana. Mobilità delle persone e dei popoli, dinamiche migratorie, emigrazioni ed immigrazioni nelle province occidentali dell'Impero romano, vol. II, Roma, Carocci editore, 2006, pp. 1337-1349; Lefebvre, Sabine, "Les migrations des Africani en Péninsule Ibérique: quelle vérite?", en Caballos Rufino, Antonio y Ségolène Demougin (eds.), Migrare. La formation des élites dans l'Hispanie Romaine, Burdeos, De Boccard, 2006, pp. 101-203, https://doi.org/10.4000/books.ausonius.7605; Ortiz Córdoba, José, “Africani en las colonias romanas de la Hispania Citerior (siglos I-II d.C.)", en Cartagine. Studi e Ricerche, 4 (2019), pp. 1-22.

${ }^{9}$ García y Bellido, Antonio, "El elemento forastero... op. cit., pp. 131-144; Haley, Evan W., Foreigners in... op. cit., pp. 152-162; Haley, Evan W., Migration and... op. cit., pp. 37-44; Gascó Lacalle, Fernando, "Presencias griegas en el sur de la península ibérica desde la época helenística al tiempo de los Severos”, en González Román, Cristobal (ed.), La sociedad de la Bética. Contribuciones para su estudio, Granada, Universidad de Granada, 1994, pp. 211-239; Gallego Franco, Henar, "Pannonios en Hispania romana”, en Hispania Antiqua, 21 (1997), pp. 341-362; Beltrán Fortes, José, "Greco-orientales en la Hispania republicana e imperial a través de las menciones epigráficas", en De Hoz, Ma Paz y Gloria Mora (eds.), El Oriente griego en la Península Ibérica: epigrafía e historia, Madrid, Real Academia de la Historia, 2013, pp. 185-204.

${ }^{10}$ García y Bellido, Antonio, "El elemento forastero... op. cit., pp. 137-139; Haley, Evan W., Foreigners in... op. cit., pp. 154-155; Haley, Evan W., Migration and... op. cit., p. 39. 
Nuestra contribución pretende ayudar a superar ese vacío. Para ello, presentamos un trabajo donde abordamos el estudio de los inmigrantes de origen tracio llegados a la península ibérica. A través del análisis de la documentación epigráfica trataremos de determinar las ciudades o pueblos de origen de estos inmigrantes, las causas que motivaron su desplazamiento y el papel que jugaron en Hispania. Prestaremos igualmente atención a otros elementos como el status social, la naturaleza de las inscripciones reunidas o las peculiaridades onomásticas que presentan los personajes estudiados.

\section{Estudio de la documentación}

La documentación epigráfica disponible en estos momentos, que constituye nuestra principal fuente de información, revela que, en el contexto general de las relaciones económicas y de movilidad establecidas entre las provincias del Imperio, la comunicación entre Hispania y Tracia fue bastante modesta y se produjo en un único sentido. Este hecho se refleja claramente en la ausencia de hispanos en esta provincia y en el escaso volumen de la inmigración tracia documentada en la península ibérica. Conviene reseñar, no obstante, que el registro epigráfico es siempre parcial y está sujeto a una continua ampliación y reinterpretación que, llegado el caso, podría variar algunas de las reflexiones recogidas en este trabajo.

Para la recopilación de la información epigráfica hemos empleado esencialmente dos criterios:

a) La mención explícita en las inscripciones estudiadas de una origo de carácter foráneo.

Este término hace referencia a la comunidad cívica o entidad territorial a la que pertenece un individuo y generalmente es diferente a la del lugar de hallazgo de su inscripción. La mención de la origo indica el interés de la persona que la emplea por conservar una cierta vinculación con su patria de origen, con la que probablemente siguiera manteniendo importantes lazos afectivos y, en algunos casos, también económicos y sociales ${ }^{11}$.

b) La presencia en la documentación revisada de nomina y cognomina de escasa implantación en la península ibérica. A partir de ellos podemos plantear la procedencia foránea de sus portadores $\mathrm{y}$, en algunos casos, su vinculación con Tracia. No obstante, es importante indicar que este criterio debe ser empleado con prudencia al no resultar tan preciso como la indicación de la origo.

\footnotetext{
${ }^{11}$ Sobre la origo ver Lassère, Jean-Marie, Manuel d'Épigraphie Romaine, París, Picard, 2005, pp. 128-136; Grüll, Tibor, "Origo as identity factor in Roman epitaphs", en Cupcea, George y Rada Varga (eds.), Social Interactions and Status Markers in the Roman World, Oxford, Archaeopress, 2018, pp. 139-150.
} 
En otras ocasiones, para la realización de estudios similares a este, hemos empleado un tercer criterio basado en la presencia de tribus poco frecuentes en Hispania. La tribu conformaba el elemento jurídico/administrativo mediante el que los ciudadanos quedaban inscritos en el censo de su civitas. Por ello, cuando en una ciudad hispana encontramos una tribu que no se corresponde con la de sus habitantes, tendemos a identificar al individuo que la porta con un inmigrante, ya sea de origen hispano o extrapeninsular. Esta metodología, que resulta bastante certera gracias a la sistematización de las tribus hispanas realizadas en diversas contribuciones $^{12}$, no es útil para este trabajo, puesto que ninguno de los inmigrantes tracios que hemos documentado presenta adscripción tribal.

Tomando como base los parámetros mencionados, hemos revisado tanto los índices de $C I L$ II, con sus diversas actualizaciones conventuales $\left(C I L \mathrm{II}^{2} / 5, C I L \mathrm{II}^{2} / 7\right.$ y $\left.C I L \mathrm{II}^{2} / 14\right)$, como los distintos corpora provinciales de la península ibérica. La documentación reunida mediante este procedimiento ha sido completada con la información incluida en publicaciones especializadas de carácter periódico, como Hispania Epigraphica (HEp) o Année épigraphique $(A E)$, y en bases de datos de tipo informático, como Epigraphik-Datenbank Clauss-Slaby (EDCS), Epigraphic Datenbank Heidelberg (EDH) o Hispania Epigraphica Online (HEpOL).

A partir de estas fuentes de información hemos elaborado un corpus epigráfico compuesto por siete inscripciones que aluden a diez personajes. Para llevar a cabo su estudio, estos individuos han sido distribuidos en dos grupos: por un lado, los inmigrantes cuyo origen tracio resulta indiscutible; por otro, aquellos individuos cuya vinculación con Tracia presenta mayores dudas. Dentro de cada grupo los personajes estudiados han sido dispuestos en orden alfabético. Dado que en la lista que presentamos se combinan nombres únicos con tria nomina, agnomina y estructuras bimembres, en los dos apartados de nuestro trabajo hemos situado en primer lugar a los individuos con tria nomina, ordenados en función de su nomen; posteriormente, aparecen dispuestos el resto de personajes en función de sus cognomina.

\subsection{Inmigrantes cuyo origen tracio podemos determinar con seguridad}

En este primer apartado hemos incluido las inscripciones de cinco individuos cuya origo podemos vincular con total seguridad con la región de Tracia.

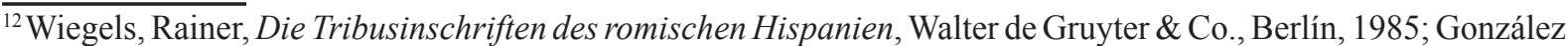
Fernández, Julián, "Urso, ¿tribu Sergia o Galeria?, en González Fernández, Julián (ed.), Estudios sobre Urso Colonia Iulia Genetiva, Sevilla, Alfar, 1989, pp. 133-154; Stylow, Armin U., "Apuntes sobre las tribus romanas en Hispania", en Veleia, 12 (1995), pp. 105-123; Fasolini, Donato, Le tribu romane della Hispania Tarraconensis. L’ascrizione tribale dei cittadini romani nelle testimonianze epigrafiche, Milán, Vita e Pensiero, 2012.
} 


\subsubsection{Marcus Aurelius Victor}

Su inscripción fue encontrada en 1913 durante la realización de unas obras en la Real Colegiata de San Isidoro de León, en cuyo museo se conserva actualmente. Se trata de una lápida funeraria que ha adquirido forma de paralelepípedo tras ser reutilizada como sillar ${ }^{13} \cdot M$. Aurelius Victor sirvió como miles durante 20 o 25 años en la legio VII Gemina. Tras su muerte, acaecida cuando contaba con 45 años, fue enterrado en Legio, campamento permanente de su unidad en Hispania. Como consecuencia de su mal estado de conservación, la pieza presenta problemas de lectura en las líneas donde figura la origo. Esta fue reconstruida inicialmente por G. Forni como natio(ne) Sax[o], lo que implicaba asignar una procedencia germana a este personaje ${ }^{14}$. Su propuesta fue seguida por otros autores ${ }^{15}$, aunque la mayor parte de la historiografía se decanta actualmente por restituir la origo de este legionario como natio(ne) [Th]rax, asignándole, en consecuencia, un origen tracio ${ }^{16}$.

En lo que se refiere a la cronología, aunque la inscripción ha sido fechada por algunos autores en el siglo II d. C. ${ }^{17}$, parece más correcto ubicarla en la primera mitad del siglo III d. C. ${ }^{18}$. Esta última fecha encajaría mejor con el epíteto Pia que ostenta la legio VII. Este apelativo le fue concedido en el año 197 por el emperador Septimio Severo y conforma de esta manera un claro terminus post quem, ya que tras este momento la legión suele aparecer en las inscripciones con el título completo de Gemina Pia Felix ${ }^{19}$. Esta circunstancia permite suponer que el reclutamiento de M. Aurelius Victor habría tenido lugar durante la dinastía de

\footnotetext{
$\overline{{ }^{13} A E \text { 1928, 173: D }}$ (is) M(anibus) s(acrum) / M(arcus) Aurel(ius) Victor / [m(iles)] leg(ionis) VII G(eminae) P(iae) F(elicis) natio(ne) / [Th]rax anno(rum) XLV st(ipendiorum) XX / opt(io) eqq(uitum) L[---] / p(edes) VIIII a[---]

${ }^{14}$ Forni, Giovanni, Il reclutamento delle legioni da Augusto a Diocleziano, Roma, Bocca, 1953, p. 227.

${ }^{15}$ Entre ellos se encuentra García y Bellido, Antonio, "El elemento forastero... op. cit., p. 133, quien asignó a $M$. Aurelius Victor una procedencia sajona (natione Saxo). Esta origo aparece también en Diego Santos, Francisco, Inscripciones Romanas de la Provincia de León (IRPLE), León, Institución Fray Bernardino de Sahagún, 1986, inscripción $\mathrm{n}^{\mathrm{o}}$ 149, aunque posteriormente fue corregida en Rabanal Alonso, Manuel A. y Sonia Ma . García Martínez, Epigrafía romana de la provincia de León: revisión y actualización (ERPLE), León, Universidad de León, 2001, inscripción $\mathrm{n}^{\circ} 129$. De forma reciente también aparece referida en la entrada que esta inscripción tiene en la base de datos Epigraphische Datebank Heidelberg bajo la referencia HD023842.

${ }^{16}$ Le Roux, Patrick, L'armée romaine et l'organisation des provinces iberiques d'Auguste a l'invasion de 409 , París, De Boccard, 1982, pp. 232 y 330; Haley, Evan W., Foreigners in... op. cit., pp. 154-155; Haley, Evan W., Migration and... op. cit., p. 39; ERPLE, 129; Palao Vicente, Juan José, Legio VII Gemina (Pia) Felix. Estudio de una legión romana, Salamanca, Universidad de Salamanca, 2006, pp. 132 y 176; Hernández Guerra, Liborio, "Veterani et milites alieni in Hispania", en Aquila Legionis, 9 (2007), p. 51, n 10; Santos Yanguas, Narciso, "Soldados legionarios de origen astur en el ejército romano", en Studia Zamorensia, 10 (2011), p. 201. No faltan otras propuestas como la formulada en los años ochenta por Rabanal Alonso, Manuel A., "La Legio VII: mandos y soldados", en Studia Historica. Historia Antigua, 6 (1988), p. 149, n' 3, corregida posteriormente (ERPLE, 129), de considerar a este personaje originario de la ciudad de Sexi (Natione Saxetana), la actual Almuñécar, o la enunciada por Roldán Hervás, José Manuel, Hispania y el ejército romano, Salamanca, Universidad de Salamanca, 1974, p. 312, n 654, para quien M. Aurelius Victor procedería de la ciudad norteafricana de Saxum Fabr.

${ }^{17}$ Rabanal Alonso, Manuel A., "La Legio VII... op. cit., p. 149, n 3; ERPLE, 129; Santos Yanguas, Narciso, "Soldados legionarios... op. cit., p. 200.

${ }_{18}^{18}$ García y Bellido, Antonio, "El elemento forastero... op. cit., p. 133; Le Roux, Patrick, L'armée... op. cit., pp. 230 y 233; Palao Vicente, Juan José, Legio VII Gemina... op. cit., p. 176.

${ }^{19}$ Rodríguez González, Julio, Historia de las legiones romanas, Madrid, Signifer Libros, 2001, pp. $250-251$.
} 
los Severos ${ }^{20}$. Es posible que también en este mismo periodo hubiese accedido a la ciudadanía romana, quizás en época de Caracalla, como reflejan su praenomen y su nomen ${ }^{21}$.

El servicio de un personaje tracio en una unidad tan vinculada a Hispania como era la VII Gemina debe relacionarse con los cambios en la composición de esta legión detectados por J.J. Palao Vicente durante el siglo III d. C., periodo en que el reclutamiento de la VII Gemina parece abrirse a regiones y gentes del Imperio que hasta ese momento no se habían caracterizado por su alto grado de romanización ${ }^{22}$.

\subsubsection{Iulius Longinus Doles}

En Calahorra (La Rioja), la antigua Calagurris, fue encontrada en 1788 la inscripción de Iulius Longinus Doles ${ }^{23}$. Apareció en la zona del Paseo del Mercadal, hacia la parte meridional del circo romano y durante años se conservó empotrada en el antiguo ayuntamiento, aunque actualmente se encuentra perdida. Conformaba una estela funeraria con una cabecera semicircular donde fue esculpida la figura de un jinete que portaba en su mano izquierda un broquel y en la derecha una lanza corta con la que atravesaba a un hombre caído; el caballo apoyaba sus patas sobre un pequeño podio similar a la base de una estatua ${ }^{24}$. La inscripción fue dedicada por Sulpicius Susulla y Fuscus Bitius en memoria de Iulius Longinus Doles. De él sabemos que pertenecía al pueblo tracio de los besos y que su llegada a la península ibérica fue consecuencia de su servicio en el ejército romano, que abarcó un periodo de 22 años.

El nombre de su unidad ha generado diferentes interpretaciones. Desde la lectura realizada por E. Hübner (CIL II, 2984) su nomenclatura fue restituida como ala Tauto(rum) Vic(trix) C(ivium) $R$ (omanorum), considerándose durante largo tiempo como el único testimonio conocido de esta unidad auxiliar ${ }^{25}$. A partir de la onomástica de los tres individuos documentados en la inscripción, A. García y Bellido consideró que el ala Tautorum sería un cuerpo de origen tracio, llegando incluso a plantear que la unidad hubiese formado parte de los auxilia de la VI Victrix en Hispania ${ }^{26}$. Se basaba para ello en el hallazgo en Calagurris de una inscripción relativa a esta legión (CIL II, 2983). Su hipótesis fue continuada por J. M. Roldán, que asumió sus mismos

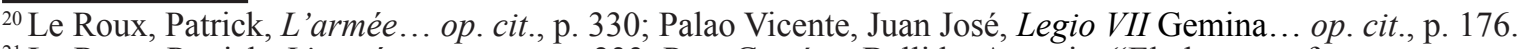

${ }^{21}$ Le Roux, Patrick, L'armée... op. cit., p. 233. Para García y Bellido, Antonio, "El elemento forastero... op. cit., p. 133, la onomástica de este legionario debería relacionarse con Marco Aurelio o Cómodo, bajo cuyos reinados consideraba que habría sido reclutado.

${ }^{22}$ Palao Vicente, Juan José, Legio VII Gemina... op. cit., pp. 131-132.

${ }^{23}$ CIL II, 2984: Iulius Lon/ginus Doles / Biticenti f(ilius) Bes/sus eques alae / Tautor(um) Vic(tricis) c(ivium) R(omanorum) f(idelis?) / an(norum) XL aer(orum) XXII h(ic) s(itus) e(st) / Sulpicius Susulla / et Fuscus Bitius / $h$ (eredes) ex t(estamento) f(aciendum) c(uraverunt.

${ }^{24}$ Esta imagen representaría, a juicio de San Vicente, José Ignacio, "Galba, el Ala Tauriana y el Ala Sulpicia", en Hispania Antiqua, 31 (2007), p. 92, al dios Caballero-Héroe, divinidad principal de los tracios.

${ }^{25}$ Sobre la misma ver Roldán Hervás, José Manuel, Hispania y el... op. cit., pp. 215-216; Le Roux, Patrick, L'armée... op. cit., pp. 87-88.

${ }^{26}$ García y Bellido, Antonio, "El Exercitus Hispanicus", Archivo Español de Arqueología, vol. 34, 103-104, 1961, pp. $135-136$.
} 
planteamientos ${ }^{27}$. De ella se desprendería que el ala Tautorum habría servido en Hispania con anterioridad al año 69-70 d. C., momento en que la VI Victrix abandonó la península ibérica ${ }^{28}$. Por su parte, P. Le Roux, aunque no dudaba de la presencia del ala Tautorum en Hispania, encuadraba su estancia con posterioridad al año $68 \mathrm{~d}$. C. Consideraba que el título honorífico de civium Romanorum exhibido por la unidad y la presencia en la inscripción de un Sulpicius permitirían llevar la pieza hasta época flavia ${ }^{29}$.

La primera variación importante en la lectura de esta inscripción la encontramos en la obra de P. A. Holder, que desarrolló el nombre de la unidad como ala Tau(riana) Tor(quata) Vic(trix) c(ivium) R(omanorum), vinculándola de esta forma con otro cuerpo ya conocido, el ala Gallorum Tauriana ${ }^{30}$. Esta lectura ha sido también recogida en varios trabajos recientes que conforman los últimos aportes sobre la trayectoria histórica de esta unidad ${ }^{31}$. Es posible que su presencia en Hispania se remontase a un momento temprano, aunque resulta más destacable su participación en los acontecimientos desencadenados tras la muerte de Nerón. En ese contexto habría respaldado inicialmente la causa de Galba y posteriormente la de Vitelio, siendo enviada más tarde por Vespasiano a la frontera del Rhin para reprimir la revuelta de Iulius Civilis, acción en la que pudo haber obtenido alguno de los cognomina que adornan su nomenclatura, particularmente los relativos a su valentía en el combate (torquata y victrix). Es posible que en este momento sus miembros hubiesen sido también recompensados con la ciudadanía romana como reconocimiento a su valor (civium Romanorum) ${ }^{32}$. Tras el fin de la revuelta el emperador Vespasiano la trasladó a Hispania, donde habría llegado entre los años 70 y 75 d. C., periodo en el que ya ostentaría los títulos honoríficos anteriormente mencionados ${ }^{33}$.

En este complejo contexto debió desarrollarse el servicio de Iulius Longinus Doles en este ala Tau(riana). Su inscripción debe fecharse con posterioridad a la llegada de la unidad a la península ibérica (70-75 d. C.) y con anterioridad a su traslado a la Mauretania Tingitana (88 d. C.), lo que permitiría situar su reclutamiento, teniendo en cuenta los 22 años de servicio consignados, a mediados del siglo i d. C. ${ }^{34}$. Doles falleció a los 40 años de edad, probablemente mientras se encontraba en activo, y recibió sepultura de manos de sus herederos y seguramente compañeros de armas, Sulpicius Susulla (2.2.4) y Fuscus Bitius (2.2.3). Su onomástica carece

\footnotetext{
${ }^{27}$ Roldán Hervás, José Manuel, Hispania y el... op. cit., p. 215.

${ }^{28}$ Rodríguez González, Julio, Historia de las... op. cit., p. 221.

${ }^{29}$ Le Roux, Patrick, L'armée... op. cit., pp. 88 y 216, n⿳ 157 . También Haley, Evan W., Foreigners in... op. cit., p. 154; Migration and... op. cit., p. 39, sitúa esta inscripción en época flavia, mientras que Roldán Hervás, José Manuel, Hispania y el... op . cit., p. 215, la adscribe de forma genérica a la segunda mitad del siglo I d. C.

${ }^{30}$ Holder, Paul A., Studies in the Auxilia of the Roman Army from Augustus to Trajan, Oxford, BAR International Series, 1980, p. 275, n 452, https://doi.org/10.30861/9780860540755. El nombre completo de esta unidad sería el de ala I Flavia Gallorum Tauriana Torquata Victrix Civium Romanorum (CIL XVI, 169, 173, 181).

${ }^{31}$ Christol, Michel y Patrick Le Roux, "L'aile Tauriana Torquata et les relations militaires de l'Hispania et de la Maurétanie tingitane entre Claude et Domitien", en Antiquités Africaine, 21 (1985), pp. 15-33, https://doi. org/10.3406/antaf.1985.1108; San Vicente, José Ignacio, “Galba, el ala... op. cit., p. 99-103. Aparece asimismo recogida en las bases de datos informáticas HEpOL (8818) y Clauss Slaby (EDCS-05502383).

${ }^{32}$ Holder, Paul A., Studies in the... op. cit., pp. 32, 36 y 39.

${ }^{33}$ Christol, Michel y Patrick Le Roux, "L’aile Tauriana... op. cit., p. 19; San Vicente, José Ignacio, “Galba, el ala... op. cit., p. 103.

${ }^{34}$ Ibídem, p. 20, nota 39.
} 
de praenomen y en ella destaca el gentilicio Iulius, que aparece acompañado de dos cognomina, el primero de ellos, Longinus, de origen latino y el segundo, tradicionalmente leído como Doles, pero para el que también poseemos la variante Dolens, de ascendencia indígena ${ }^{35}$. Este cognomen ha sido considerado por J. I. San Vicente como el nombre tracio de nuestro protagonista ${ }^{36}$. Sin embargo, ni las inscripciones empleadas por este autor como ejemplo ni el resto del registro epigráfico de este apelativo justifican su afirmación, ya que la vinculación del cognomen Doles con Tracia se constata únicamente en $A E$ 1929, 211, donde figura la correspondiente origo. Igualmente limitado es el registro epigráfico de la variante Dolens, cuya relación con Tracia solo aparece explicitada en $A E$ 1977, 722 y quizás podría suponerse en CIL III, 6255.

En relación al status jurídico de Doles podríamos plantear la posibilidad de que, dado sus años de servicio, hubiese participado en la lucha contra Civilis, donde habría recibido la ciudadanía romana junto al resto de su unidad. Sin embargo, resultaría sorprendente que alguien que hubiese accedido a la ciudadanía romana en época de Vespasiano eligiese el nomen Iulius en lugar del gentilicio Flavius, vinculado al emperador que le otorgó dicho privilegio. Por ello, parece más lógico pensar que Doles hubiese sido ciudadano con anterioridad. Esta circunstancia contrastaría con su servicio en una cohorte auxiliar, un hecho poco común pero que cuenta con algunos paralelos, como ha recogido P. A. Holder $^{37}$.

\subsubsection{Fuscus Dorilsis}

Su inscripción fue encontrada en 1905 en Astorga (León) durante un derribo en la muralla sur de la ciudad, donde había sido reutilizada para cubrir una cloaca. Actualmente se conserva en el Museo de los Caminos, ubicado en dicha localidad. Conforma una estela funeraria cuya cabecera semicircular está decorada con una roseta o florón hexapétalo en relieve cuyas hojas parten de un botón central. En ella se recoge el epitafio de Fuscus Dorilsis, originario de Serdica (domo Serdus), ciudad que debemos identificar con la actual Sofía (Bulgaria) ${ }^{38}$. Su procedencia quedaría reflejada igualmente a través de su filiación, ya que el nombre de su padre, Eptaecentus, aunque poco frecuente, figura en la onomástica de un individuo de origen tracio documentado en un diploma militar de Mogontiacum (Mainz, Alemania) ${ }^{39}$. La onomástica de Fuscus Dorilsis carece de praenomen y conforma un buen ejemplo de estructura onomástica de doble idiónimo. En ella conviven un cognomen latino, Fuscus, y otro indígena, Dorilsis, quizás el antiguo nombre tracio de este personaje, un extremo que no podemos confirmar al

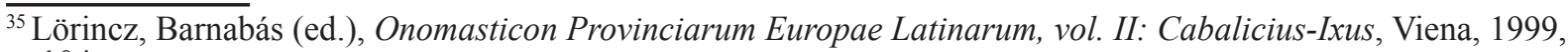
p. 104.

${ }^{36}$ San Vicente, José Ignacio, "Galba, el ala... op. cit., p. 95 y nota 34.

${ }^{37}$ Holder, Paul A., Studies in the... op. cit., pp. 49-50.

${ }^{38}$ ERPLE, 82: Fuscus Dori/lsis / Eptaece/nti f(ilius) miles ex / cohorte T(h)rac/um |(centuria) Iul(i) Martial(is) / dom(o) Serdus ann(orum) / XXV aer(orum) VIIII h(ic) s(itus) e(st).

${ }^{39}$ García y Bellido, Antonio, "El elemento forastero... op. cit., p. 137; Le Roux, Patrick, L'armée... op. cit., p. 89. La inscripción mencionada es CIL XIII, 6821: Mucapori Eptacentis f. Thrac.
} 
no contar con ninguna otra referencia epigráfica de este $\operatorname{cognomen}^{40}$. Todo ello indicaría que, probablemente, nos encontramos ante un peregrinus que habría latinizado parcialmente su onomástica. En este ámbito su caso presenta importantes similitudes con el de Fuscus Bitius (2.2.3).

Dorilsis falleció en Asturica Augusta con 25 años y tras haber servido durante nueve en una cohors Thracum. Estas cifras tan bajas indicarían que su muerte habría tenido lugar mientras se encontraba en activo. Su unidad formaba parte de los cuerpos auxiliares del ejército romano, aunque la ausencia de numeral en su nomenclatura dificulta su identificación. Para A. García y Bellido y J. M. Roldán Hervás pudo tratarse de la cohors IIII Thracum equitata, unidad que habría formado parte del ejército con el que Augusto culminó la conquista del norte peninsular y cuya estancia en Hispania ponían en relación con la de la legio IV Macedonica ${ }^{41}$. Junto a ella pudo haber abandonado la Península en el año 39-40 para instalarse en el limes renano, donde conocemos la presencia en época julio-claudia de una cohors IV Thracum en Germania Inferior $^{42}$. Contrario a esta propuesta se mostró P. Le Roux, para quien los argumentos esgrimidos no permitirían asimilar la cohors IIII nombrada en la inscripción de Fuscus Dorilsis con la cohors IV Thracum documentada en Germania. En su lugar, planteaba la posibilidad de que se tratase de una unidad diferente de la que solo conocemos esta inscripción. Dicha tropa, compuesta fundamentalmente por tracios, habría llegado a la península ibérica en época de Claudio $^{43}$, periodo en el que la mayoría de los autores ha situado esta inscripción ${ }^{44}$.

La presencia de Fuscus Dorilsis en Asturica Augusta podría relacionarse con el rango de capital conventual ostentado por esta ciudad, que pudo haber conformado durante un tiempo la residencia de uno de los legados del gobernador provincial. Por tanto, sería posible vincular algunas de las inscripciones de militares allí encontradas, entre ellas la de Dorilsis, con la actividad desarrollada por este legado ${ }^{45}$. No obstante, tampoco debe obviarse la posible vinculación de la cohors Thracum con la legio X Gemina, cuyo campamento principal se encontraba a cuarenta kilómetros de Asturica $^{46}$.

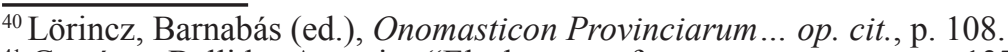

${ }^{41}$ García y Bellido, Antonio, "El elemento forastero... op. cit., p. 137, nota 1; Roldán Hervás, José Manuel, Hispania y el... op. cit., p. 223. Ambos autores realizaron esta identificación a partir de las inscripciones de dos prefectos que comandaron esta unidad encontradas en Tarraco $\left(C I L \mathrm{II}^{2} / 14,1010\right.$ y 1132).

${ }^{42}$ Roldán Hervás, José Manuel, Hispania y el... op. cit., p. 223.

${ }^{43}$ Le Roux, Patrick, L'armée... op. cit., pp. 89-90.

${ }^{44}$ Le Roux, Patrick, L'armée... op. cit., p. 90; Haley, Evan W., Foreigners in... op. cit., p. 154; Haley, Evan W., Migration and... op. cit., p. 39. Una cronología similar se ofrece en EAstorga, 30 e ILAstorga,40, donde toman como referencia el año 39-40 d. C., momento en que la IIII Macedonica, unidad con la que vinculan a la cohors IIII Thracum, abandonó Hispania. Por su parte, Roldán Hervás, José Manuel, Hispania y el... op. cit., p. 342, nº 478 , la sitúa de forma genérica en el siglo i d. C., mientras que en ERPLE, 171 la fechan en un momento avanzado el siglo i d. C.

${ }^{45}$ Le Roux, Patrick, L’armée... op. cit., p. 122; Ortiz Córdoba, José, “De Gallia a... op. cit., pp. 165-168.

${ }^{46}$ Palao Vicente, Juan José, "La Res Militaris en Hispania durante la época de la Naturalis Historia de Plinio", en Ciprés, Pilar (ed.), Plinio el Viejo y la construcción de la Hispania Citerior, Vitoria, Universidad del País Vasco, 2017, p. 172.
} 
Del territorio de Ossigi Latonium (Cerro Alcalá, Jaén) procede la estela funeraria de un joven esclavo llamado Nusatita ${ }^{47}$. La inscripción fue encontrada en las cercanías de Albánchez (Jaén), en un lugar denominado "El Campanil”, aunque actualmente se conserva en el Museo Arqueológico Nacional. Su cronología fue fijada inicialmente por J. Mangas y C. González Román en época de Trajano (CILA III, 351). Sin embargo, en la revisión de la pieza realizada por el propio C. González en $C I L I^{2} / 7,24$ se ha optado por llevar la cronología hasta el siglo III d. C.

La mencionada estela fue colocada en la tumba de Nusatita tras su prematura muerte, acaecida cuando contaba con más o menos tres años de edad. El texto epigráfico lo define como puer serv(a)e pronatus natione Tracie. A través de esta expresión podemos constatar su carácter esclavo (puer serv(a)e) y también su ascendencia tracia (pronatus natione Tracie). En ella destaca el empleo del término pronatus, seguramente una mala transcripción del correcto prognatus, con el significado de "descendiente" u "oriundo de", como señalaron J. Mangas y C. González Román (CILA III, 351). Este hecho, unido a su escasa edad, permite suponer que la verdadera emigrante habría sido su madre, de nombre desconocido pero seguramente también de origen tracio (2.2.6). Desde esta región se habría desplazado, suponemos que no de forma voluntaria dado su status servil, hasta la localidad bética de Ossigi. A partir de ahí se abren dos posibilidades en relación con Nusatita. Por un lado, que hubiera nacido en Tracia y llegado con su madre a la Bética, opción complicada, aunque no improbable, si pensamos en los enormes riesgos que implicaría la realización de un viaje tan largo con un niño tan pequeño; por otro, que hubiese nacido ya en territorio hispano, por lo que estaríamos realmente ante un descendiente de inmigrantes, de tal modo que la presencia en su inscripción de la expresión natione Tracie pudo haber tenido como objetivo mantener el recuerdo y la vinculación sentimental con la patria de origen.

Con relación a la onomástica de Nusatita debemos reseñar su carácter sumamente simple, como corresponde a la población de ámbito servil. Su nombre carece de paralelos en la epigrafía latina, por lo que se ha planteado la posibilidad de que se trate de un apelativo de origen hispano ${ }^{48}$.

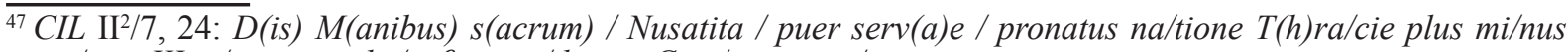
anno/rum III ia/cet petito be/neficio in / locum Cam/panianen/sem.

${ }^{48}$ Albertos Firmat, $\mathrm{M}^{\mathrm{a}}$. Lourdes, La onomástica personal primitiva de Hispania, Salamanca, Consejo Superior de Investigaciones Científicas, 1966, p. 171.
} 
2.1.5. ¿Simp(lex)?

En Cádiz fue encontrado en el siglo XVII el epitafio de un gladiator del tipo hoplomachus que murió en esta ciudad durante el siglo i d. C.49. La inscripción, actualmente perdida, presenta problemas de lectura en su primera línea, donde la mayor parte de los autores consideran que habría figurado el nombre corrompido de este gladiador. Quizás pudiera tratarse de Simp(lex) o Simp[le]cs, aunque quienes han abordado el estudio de la inscripción toman este nombre con prudencia o directamente lo rechazan ${ }^{50}$. Junto a la onomástica se encuentra la abreviatura $L E R T$, de difícil interpretación. Inicialmente, A. García y Bellido y P. Piernavieja consideraron que podría tratarse de una mala lectura del término LIBER(atus), una expresión que indicaría que este gladiador ya no estaba sometido a la disciplina de un ludus y que, por tanto, podía alquilar sus servicios a cualquier editor que quisiera contratarlos ${ }^{51}$.

Esta propuesta ha sido descartada por otros autores posteriores, como F. Gascó, para quien estas letras podrían hacer referencia a la condición libertina de este gladiador, o A. Ceballos, que piensa que en esta línea podría restituirse el término VET(eranus) en lugar de la abreviatura $L E R T^{52}$. También J. Gómez-Pantoja considera improbable la hipótesis planteada por A. García y Bellido y P. Piernavieja, ya que la forma habitual de expresar la manumisión solía ser lib(er) o l(iber), por lo que no descarta que LERT pudiera ser realmente una mala lectura del agnomen ${ }^{53}$.

Estas limitaciones no nos impiden conocer otros datos de la trayectoria vital de este luchador, como su edad de fallecimiento, 35 años, o su número de victorias, 20. Tras su muerte fue su esposa, cuyo nombre no figura en el texto, la encargada de levantar el monumento funerario, donde se indicó el origen de su cónyuge: natione Bessus. Gracias a esta referencia sabemos que este gladiator perteneció al pueblo de los besos, que habitaban en la Tracia central. Su muerte en Gades pudo haber sido consecuencia de algún combate ${ }^{54}$. De ser cierta dicha hipótesis podríamos suponer que este hoplomachus habría llegado hasta esta ciudad de la Bética formando parte de las familiae gladiatoriae alquiladas para participar en los espectacula

\footnotetext{
${ }^{49}$ CIL II, 1739: Simp(lex) C(ai?) s(ervus) LERT / (h)opl(omachus) palmaru(m) / XX natione / Bessus an(norum) $X X X V /$ ucsor viro b(ene) m(erenti).

${ }^{50} \mathrm{Al}$ respecto ver Piernavieja, Pablo, Corpus de inscripciones deportivas de la España romana, Madrid, Instituto Nacional de Educación Física, 1977, p. 152; Ceballos Hornero, Alberto, Los espectáculos en la Hispania romana: la documentación epigráfica, Cuadernos Emeritenses 26, Mérida, 2004, p. 490; Gómez-Pantoja, Joaquín, Epigrafia anfiteatrale dell'occidente romano, VII. Baetica, Tarraconensis, Lusitania, Roma, Quasar, 2009, p. 103. Por su parte, García y Bellido, Antonio, "El elemento forastero... op. cit., p. 137 y "Lapidas funerarias de gladiadores de Hispania”, Archivo Español de Arqueología, vol. 33, 101-102 (1960), p. 139, n 12, consideró que el nombre de este gladiador era ilegible.

${ }^{51}$ García y Bellido, Antonio, "Lapidas funerarias de..." op.cit.,p. 139; Piernavieja, Pablo, Corpus de inscripciones... op. cit., p. 152.

${ }^{52}$ Gascó Lacalle, Fernando, “Presencias griegas en...”op. cit., p. 234;Ceballos Hornero, Alberto, Los espectáculos en la...op. cit., p. 490.

${ }^{53}$ Gómez-Pantoja, Joaquín, Epigrafia anfiteatrale... op. cit., p. 104.

${ }^{54}$ García y Bellido, Antonio, "El elemento forastero..."op. cit.p. 137.
} 
locales; no obstante, también existe la posibilidad de que fuese un esclavo tracio formado como gladiador en el supuesto ludus bético ${ }^{55}$.

Tabla 1: Inmigrantes de origen tracio documentados en Hispania.

\begin{tabular}{|c|c|c|c|c|c|c|}
\hline Onomástica & Cron. & Origo & $\begin{array}{l}\text { Lugar del } \\
\text { hallazgo }\end{array}$ & Status & Función Social & Referencia \\
\hline $\begin{array}{c}\text { Marcus } \\
\text { Aurelius Victor }\end{array}$ & Siglo III d. C. & $\begin{array}{l}\text { Natio(ne) } \\
\text { [Th]rax }\end{array}$ & Legio & $\begin{array}{c}\text { Civis } \\
\text { Romanus }\end{array}$ & $\begin{array}{c}{[\text { [m(iles)] leg(ionis) VII }} \\
G(\text { eminae) } P(\text { iae }) F(\text { elicis })\end{array}$ & $A E 1928,173$ \\
\hline $\begin{array}{l}\text { Fuscus } \\
\text { Dorilsis }\end{array}$ & Siglo I d. C. & $\begin{array}{l}\operatorname{Dom}(o) \\
\text { Serdus }\end{array}$ & $\begin{array}{l}\text { Asturica } \\
\text { Augusta }\end{array}$ & Peregrinus & Miles ex cohorte $T(h)$ racum & ERPLE, 82 \\
\hline $\begin{array}{c}\text { Iulius } \\
\text { Longinus } \\
\text { Doles }\end{array}$ & Siglo I d. C. & Bessus & Calagurris & $\begin{array}{c}\text { Civis } \\
\text { Romanus }\end{array}$ & $\begin{array}{c}\text { Eques alae Tau(rianae) } \\
\text { tor(quatae) Vic(tricis) } \\
\text { c(ivium) R(omanorum) }\end{array}$ & CIL II, 2984 \\
\hline Nusatita & Siglo III d. C. & $\begin{array}{l}\text { Natione } \\
\text { T(h)racie }\end{array}$ & Ossigi & Servus & & $C I L \mathrm{II}^{2} / 7,24$ \\
\hline ¿Simp(lex)? & Siglo I d. C. & $\begin{array}{l}\text { Natione } \\
\text { Bessus }\end{array}$ & Gades & ¿Liberatus? & Gladiator (hoplomachus) & CIL II, 1739 \\
\hline
\end{tabular}

\subsection{Inmigrantes de posible origen tracio}

En este segundo bloque hemos incluido aquellos personajes cuyo origen tracio resulta dudoso o no puede ser determinado con seguridad debido a la ausencia de origo o al mal estado de conservación de algunas inscripciones.

\subsubsection{Marcus Aurelius Achaicus}

Su inscripción fue descubierta hacia 1985 junto al Camino Viejo de Granada, unos 6 km al sudeste de Baena (Córdoba), en terrenos pertenecientes al ager del antiguo municipio romano de Iponoba. Actualmente se conserva en un domicilio particular de Baena. Se trata de un ara funeraria realizada en piedra caliza que presenta un importante desgaste y numerosas roturas que dificultan su lectura e interpretación. Fue dada a conocer por A. U. Stylow, que pudo verla personalmente y fotografiarla en el año 1990, en $C I L \mathrm{II}^{2} / 5,375^{56}$. En su comentario reseñaba las dificultades existentes para restituir correctamente las líneas 3-5 de la inscripción. Entre las consideraciones que realizó estaba la posibilidad de situar ahí la origo del personaje, que por las letras conservadas y la disposición del texto se aventuró a reconstruir como Astigit(anus). La inscripción ha sido datada en el siglo III d. C. a partir de la paleografía y de la onomástica de su protagonista.

\footnotetext{
${ }_{55}^{5}$ Piernavieja, Pablo, Corpus de inscripciones...op. cit., p. 152; Ceballos Hornero, Alberto, Los espectáculos en la...op. cit., p. 491.

${ }^{56}$ CIL II $2 / 5,375: M($ arcus) Au(relius) / Ac(h)aicu/s ISI IA(?) / CETA / V(?)[---]II / (h)ic s[it]u/s est / sit tibi t/er(ra) $l(e) v(i) s$.
} 
La lectura realizada por A. U. Stylow ha sido parcialmente rechazada por A. Canto en el comentario de la inscripción recogido en Hispania Epigráfica, donde se propone una nueva lectura de la pieza ${ }^{57}$. Según la misma, el texto contaría con dos líneas más, primera y última, donde se incluirían la dedicación a los dioses manes (Dis M(anibus) y la onomástica, parcialmente conservada, del dedicante de la pieza: [- - -] balus f(ecit). Asimismo, esta autora apuesta por precisar la edad de fallecimiento de M. Aurelius Achaicus, que fija en 23 años y un mes, y por modificar su origo, que considera debería leerse como nat(ione) Geta. Según esta hipótesis, M. Aurelius Achaicus sería un inmigrante de origen tracio, pues la origo propuesta aludiría al pueblo de los Getas, una de las tribus que habitaban junto al Danubio, en territorios que actualmente se corresponden con el norte de Bulgaria y los distritos rumanos de Munteniana y Dobruja.

Esta propuesta, aunque sugerente, debe ser tomada con prudencia, ya que la expresión nat(ione) Geta cuenta con muy pocos paralelos en la epigrafía. De hecho, solo hemos podido encontrar esta fórmula en una inscripción de Beverston (Inglaterra) ${ }^{58}$. Tampoco la onomástica de este personaje presenta especificidades propias que nos permitan vincularlo con Tracia. Al contrario, el nomen Aurelius es un gentilicio imperial muy extendido en algunas provincias danubianas tras el reinado de M. Aurelio y, sobre todo, tras la promulgación de la Constitutio Antoniniana ${ }^{59}$, mientras que Acaicus o Achaicus es un cognomen griego que presenta una limitada difusión ${ }^{60}$.

\subsubsection{Caius Valerius Avitus}

Su inscripción está compuesta por un fragmento de placa de mármol que se encuentra fracturado en sus partes superior, inferior y derecha. El campo epigráfico aparece rehundido y está rodeado por una moldura decorada con motivos vegetales. Fue encontrada en Córdoba a mediados del siglo XVIII y posteriormente donada al Museo Arqueológico Nacional, donde se conserva actualmente. En ella fue grabado el epitafio de C. Valerius Avitus ${ }^{61}$, que muestra la particularidad de ser una composición parcialmente poética, ya que al praescriptum en prosa le siguen tres versos hexamétricos conservados fragmentariamente y cuya interpretación resulta complicada $^{62}$. La pieza fue datada por A. U. Stylow entre finales del siglo il y comienzos del

\footnotetext{
$\overline{{ }^{57} H E p ~ 8,1998,146: ~ D i s ~ M(a n i b u s) ~ / ~ M(a r c u s) ~ A u r(e l i u s) ~ A c(h) a i c u / s ~[-~-~-] ~ i ~ f(i l i u s) ~ n a t(i o n e) ~ / ~ G e t a ~ / ~ v(i x i t) ~}$ a(nnos) XXIII / me(n)s(em) I p(ius) i(n) sui/s e(s?) h(ic) / s(itus) e(st) s(it) t(ibi) t(erra) l(evis) [- - -]/balus f(ecit). Esta lectura aparece recogida también en HEpOL (2275) y Clauss-Slaby (EDCS-19100787).

${ }^{58}$ RIB, 136: D(is) M(anibus) / Metti n/ation(e) / Geta / vixit / ann(os) XXXV/h(eres) p(osuit).

${ }^{59}$ Lörincz, Barnabás (ed.), Onomasticon Provinciarum Europae Latinarum, vol. I: Aba-Bysanus, Budapest, 2005 , pp. 99-105.

${ }^{60}$ Solin, Heikki, Die griechische Personenamen in Rom. Ein Namenbuch, Berlin-Nueva York, Walter de Gruyter, 1982, p. 571; Lörincz, Barnabás (ed.), Onomasticon Provinciarum ... vol. I. op. cit., p. 18.

${ }^{61}$ CIL II 2/7, 394: D(is) [M(anibus) s(acrum)] / C(aius) Val(erius) Avitus [- - -] / natione Ta [- - -] / gemma quod est AV[- - - ] / quod tenebris [- - -] / sic inter A[- - -] / - - - - -

${ }^{62}$ Para un mayor detalle remitimos al comentario de Fernández Martínez, Concepción y Remedios Carande Herrero, en Fernández Martínez, C., Carmina Latina Epigraphica de la Bética Romana. Las primeras piedras de
} 
III d. C. (CIL II²/7, 394), aunque C. Fernández Martínez y R. Carande Herrero consideran que podría remitir a un momento más tardío (CLEBetica, CO04).

En lo que a este trabajo se refiere debe reseñarse la procedencia foránea de $C$. Valerius Avitus, cuya origo confirma que no era un cives Cordubensis. Por desgracia, parte de la línea donde figura el origen de este personaje ha desaparecido, por lo que resulta complicado determinar con certeza su lugar de nacimiento. De la origo, que comienza con el término natio, se conservan únicamente una $T$ y el comienzo de una segunda letra que E. W. Haley consideró una $A$. De esta forma, restituyó la origo de Avitus como natione Ta[porus?], vinculando a este personaje con el populus lusitano de los Tapori $^{63}$. Los planteamientos de Haley fueron considerados válidos por A. U. Stylow en la revisión de esta inscripción realizada en $C I L \mathrm{II}^{2} / 7$, 394, donde también se restituye la origo como natione Ta [- - - ]. Sin embargo, en nuestro caso creemos que esta reconstrucción debería tomarse con prudencia, ya que el concepto natio alude generalmente a una entidad territorial más amplia que la conformada por un simple populus como eran los Tapori.

Por ello, C. Fernández Martínez y R. Carande Herrero han ofrecido una lectura diferente (CLEBetica, CO04). Para estos autores la letra existente tras la $T$ no sería una $A$, sino una $H$, lo que permitiría restituir la origo como natione Th [- - - ]. De ello derivaría la posibilidad de reconstruir la parte perdida del texto como Th(rax) o Th(racius), un término que sería más verosímil por las letras conservadas y encajaría mejor con el empleo del concepto natio. Esta propuesta cuenta con un paralelo cercano en la inscripción de Nusatita, cuya procedencia fue indicada mediante la fórmula natione $T(h)$ racie $\left(C I L \mathrm{II}^{2} / 7,24\right)$.

Conviene reseñar, igualmente, que el empleo del término natio seguido del nombre de la región de origen se documenta en Corduba en el epitafio de varios gladiadores. Sin embargo, la posibilidad de considerar a C. Valerius Avitus como un gladiador debería descartarse por dos razones: la primera es su tria nomina, circunstancia que aleja su onomástica de las estructuras simples que solemos encontrar en el mundo gladiatorio; la segunda es el lugar de procedencia de su inscripción, que no presenta ninguna relación con el Camino Viejo de Almodóvar, donde han aparecido prácticamente todas las inscripciones gladiatorias de Corduba.

\subsubsection{Fuscus Bitius}

La información que poseemos sobre Fuscus Bitius es muy escasa. Fue uno de los dedicantes del epitafio del eques Iulius Longinus Doles (2.1.2), lo que permite suponer que habría sido compañero de armas del difunto en el ala Tau(riana). Su posible origen tracio fue sugerido por A. García y Bellido tomando como base su cognomen, que este autor consideraba

nuestra poesía, Sevilla, Universidad de Sevilla, 2007, referencia CO04.

${ }^{63}$ Haley, Evan W., Foreigners in... op. cit., p. 229, n 320; Haley, Evan W., Migration and... op. cit., p. $66, \mathrm{n}^{\mathrm{o}} 332$. 
propio de Tracia, y su designación como heredero de Longinus Doles ${ }^{64}$. Sus planteamientos han sido seguidos en buena medida por otros autores posteriores, que han atribuido también un origen tracio a este personaje ${ }^{65}$.

La onomástica de Fuscus Bitius presenta algunas similitudes con el caso ya comentado de Fuscus Dorilsis (2.1.3). Nuevamente volvemos a ver la combinación del cognomen latino Fuscus con un segundo elemento de raíz indígena que ha sido tradicionalmente restituido como Bitius, aunque consideramos que no pueden descartase otras formas como Bithus o Bitus, que cuentan con un mayor número de testimonios en el área del bajo Danubio ${ }^{66}$. El análisis de la onomástica de este personaje plantea dos posibles interpretaciones. Por un lado, podríamos estar ante una estructura de doble idiónimo, propia de un peregrinus. Por otro, sería posible considerar también que nos encontremos ante una onomástica invertida que responda a la fórmula cognomen más nomem, de tal manera que el cognomen sería Fuscus y el gentilicio, Bitius, un esquema onomástico compatible con la ciudadanía romana o latina. Este fenómeno se documenta en varias inscripciones hispanas ${ }^{67} \mathrm{y}$, de hecho, Bitius ha sido registrado en posición de nomen en algunas ocasiones, por lo que esta opción no sería descartable ${ }^{68}$.

Estas diferentes interpretaciones tienen su correspondiente reflejo en el status jurídico del personaje. La primera posibilidad apuntaría a Bitius como un peregrinus que habría latinizado su onomástica incluyendo un término latino que habría desplazado a su antiguo nombre indígena, situado ahora como cognomen ${ }^{69}$. Esta peculiar estructura onomástica indicaría, a juicio de J.I. San Vicente, que el apelativo c(ivium) R(omanorum) otorgado al ala Tau(riana) y el acceso a la ciudadanía que este conllevaba afectaron únicamente a los miembros que componían la unidad en el momento de su concesión, mientras que aquellos que se incorporaron al ala con posterioridad, como pudo ocurrir con Bitius, habrían seguido manteniendo su status peregrino hasta el momento de su licenciamiento ${ }^{70}$. Por el contrario, si aceptamos la propuesta de una onomástica invertida podría plantearse la posibilidad de que Fuscus Bitius hubiese recibido la ciudadanía romana tras ayudar a reprimir la revuelta de Civilis. Esta interpretación encajaría con la onomástica de Sulpicius Susulla, que, como detallamos a continuación, también pudo haber sido un ciudadano romano.

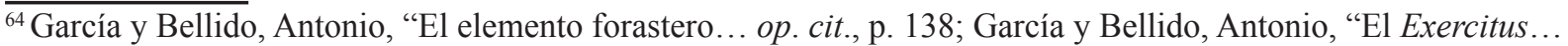
op. cit., p. 136.

${ }_{65}^{6}$ Le Roux, Patrick, L'armée...op. cit., p. 88; Haley, Evan W., Foreigners in... op. cit., p. 154; Haley, Evan W., Migration and... op. cit., p. 39; Christol, Michel y Patrick Le Roux, "L'aile Tauriana... op. cit., p. 20; San Vicente, José Ignacio, "Galba, el ala... op. cit., p. 95.

${ }^{66}$ Lörincz, Barnabás (ed.), Onomasticon Provinciarum... vol. I. op. cit., p. 121; Dana, Dan, "L'impact de l'onomastique latine sur les onomastiques indigenes dans l'espace thrace", en Dondin-Payre, Monique (ed.), Les noms de personnes dans l'Empire romain. Transformations, adaptation, évolution, Bordeaux, Ausonius Éditions, 2011, p. 54

${ }^{67}$ Álvarez Melero, Anthony y Manuel Parodi Álvarez, "Notes sur une inscription de la Baie de Cadix: l'épitaphe d'Optata Erennia", en SPAL, 19 (2010), pp. 199-200. https://doi.org/10.12795/spal.2010.i19.09

${ }^{68}$ Solin, Heikki y Olli Salomies, Repertorium nominum gentilium et cognominum Latinorum, Hildesheim, Olms-

Weidmann, 1988, p. 35; Lörincz, Barnabás (ed.), Onomasticon Provinciarum ... vol. I. op. cit., p. 121.

${ }^{69}$ San Vicente, José Ignacio, "Galba, el ala... op. cit., p. 98.

${ }^{70}$ Ibídem. Sobre esta cuestión ver, asimismo, Holder, Paul A., Studies in the ... op. cit., p. 32.
} 


\subsubsection{Sulpicius Susulla}

Sulpicius Susulla fue el segundo dedicante de la inscripción de Iulius Longinus Doles (2.1.2). Al igual que ocurre con Fuscus Bitius (2.2.3), podemos suponer que también habría sido compañero de armas de Doles en el ala Tau(riana). Su onomástica carece de praenomen y presenta, nuevamente, un elemento latino y otro indígena, aunque, a diferencia del caso anterior, tiene una estructura clara de nomen más cognomen. En ella destaca el gentilicio Sulpicius, que permite considerar la posibilidad de que nos encontremos ante un ciudadano romano. Esta es la postura defendida por J.I. San Vicente, para quien Susulla habría recibido la ciudadanía romana en tiempos de Galba tras haber culminado los 25 años de servicio reglamentarios y con anterioridad a que su unidad hubiese ganado el título de civium Romanorum en la lucha contra Civilis $^{71}$. Sin embargo, dada la escasa información que poseemos sobre el personaje, conviene ser prudentes en este sentido, ya que no conocemos sus años de servicio ni tampoco el momento en que tuvo lugar su reclutamiento, por lo que no podemos descartar que se hubiese alistado cuando el Ala Tauriana era ya una unidad de ciudadanos romanos.

El origen tracio de Susulla fue sugerido por A. García y Bellido siguiendo los mismos motivos expuestos en el caso anterior (ver. 2.2.3 y notas 64 y 65). Sin embargo, conviene tomar nuevamente con prudencia esta afirmación, ya que la designación como heredero no es por sí misma indicativa de un origen común. De igual modo, el cognomen Susulla conforma un unicum, por lo que no contamos con otros paralelos que permitan establecer una clara asociación entre este cognomen y la región de Tracia, invalidando así su empleo como un elemento indicativo del origen de este personaje.

\subsubsection{Ignota}

En última instancia nos queda por referir en este apartado el caso de la madre de Nusatita (2.1.4), cuya onomástica desconocemos. Esta mujer aparece mencionada de forma indirecta en el epitafio de este pequeño esclavo cuando el texto epigráfico subraya su status servil (puer serv(a)e). Dada las dificultades del viaje y la corta edad de Nusatita es muy posible que ella hubiese sido la verdadera itinerante ${ }^{72}$. Por ello, podemos suponer que también sería de origen tracio y que habría llegado a la Bética junto a su hijo o poco antes del nacimiento de este.

\footnotetext{
${ }_{71}^{71}$ San Vicente, José Ignacio, “Galba, el ala... op. cit., pp. 96-97.

${ }^{72}$ García y Bellido, Antonio, "El elemento forastero..."op. cit.p. 138; Mirón Pérez, Ma . Dolores, "La movilidad espacial de mujeres y su identificación en la Bética romana", Hispania Antiqua, 37-38 (2013-2014), p. 312.
} 
Tabla 2: Inmigrantes de posible origen tracio documentados en Hispania.

\begin{tabular}{|c|c|c|c|c|c|c|}
\hline Onomástica & Cron. & Origo & Domicilio & Status & Función social & Referencia \\
\hline $\begin{array}{l}\text { Marcus } \\
\text { Aurelius } \\
\text { Achaicus }\end{array}$ & Siglo III d. C. & $\begin{array}{c}\text { ¿Natione } \\
\text { Geta? }\end{array}$ & Iponoba & $\begin{array}{l}\text { ¿Libertus? } \\
/ \text { ¿Civis } \\
\text { Romanus? }\end{array}$ & & $\begin{array}{c}C I L \mathrm{II}^{2} / 5 \\
375\end{array}$ \\
\hline Fuscus Bitius & Siglo i d. C. & & Calagurris & $\begin{array}{l}\text { Peregrinus } \\
\text { / ¿Civis } \\
\text { Romanus? }\end{array}$ & $\begin{array}{c}\text { Miles ¿alae Tau(rianae) } \\
\text { tor(quatae) Vic(tricis) } \\
\text { c(ivium) R(omanorum)? }\end{array}$ & CIL II, 2984 \\
\hline $\begin{array}{c}\text { Caius Valerius } \\
\text { Avitus }\end{array}$ & $\begin{array}{c}\text { Transición siglo } \\
\text { II al III d. C. }\end{array}$ & $\begin{array}{l}\text { Natione } \\
\text { Th[- - - ] }\end{array}$ & $\begin{array}{l}\text { Colonia } \\
\text { Patricia }\end{array}$ & Civis Romanus & & $\begin{array}{c}C I L \mathrm{II}^{2} / 7 \\
394\end{array}$ \\
\hline $\begin{array}{c}\text { Sulpicius } \\
\text { Susulla }\end{array}$ & Siglo i d. C. & & Calagurris & $\begin{array}{c}\text { ¿Civis } \\
\text { Romanus? }\end{array}$ & $\begin{array}{l}\text { Miles ¿alae Tau(rianae) } \\
\text { tor(quatae) Vic(tricis) } \\
\text { c(ivium) R(omanorum)? }\end{array}$ & CIL II, 2984 \\
\hline Ignota & Siglo III d. C. & & Ossigi & Serva & & CIL II $\mathrm{II}^{2} / 7,24$ \\
\hline
\end{tabular}

\section{Conclusiones}

La presencia de individuos de origen tracio en Hispania resulta bastante modesta atendiendo a la documentación epigráfica disponible actualmente. Esta conforma un elenco limitado de inscripciones, todas ellas funerarias, que nos han permitido documentar con certeza a cinco individuos de origen tracio (tabla 1). A ellos podríamos sumar otros cinco casos donde la procedencia tracia de sus protagonistas no está totalmente clara (tabla 2). Las inscripciones estudiadas se concentran en las provincias Citerior y Baetica y han sido datadas en los siglos I y III d. C. Dentro de los individuos que podemos considerar con seguridad de origen tracio observamos que tres de ellos han sido localizados en la Hispania Citerior, concretamente en Legio, Calagurris y Asturica Augusta, mientras que los dos individuos restantes proceden de la Bética, donde han sido hallados en Gades y Ossigi.

Por el contrario, muy poco podemos decir sobre los centros emisores, ya que entre las inscripciones estudiadas solo contamos con una referencia a una ciudad concreta, en este caso Serdica (domo Serdus), mencionada en el epitafio de Fuscus Dorilsis (2.1.3). El resto de individuos identificados indican su origo empleando el término natio (ablativo natione) seguido de una referencia geográfica o étnica. Esta puede aludir a la propia región de Tracia, como ocurre con M. Aurelius Victor (2.1.1) y Nusatita (2.1.4), que emplean las fórmulas Natio(ne) [Th] rax y Natione T(h)racie, respectivamente, o bien hacer referencia a algunos de los pueblos que habitaban en esa provincia, como los besos en los casos de Iulius Longinus Doles (2.1.2) y ¿Simp(lex)? (2.1.5) o los getas, siempre que aceptemos la propuesta de una procedencia 
tracia para M. Aurelius Achaicus (2.2.1). Esta forma de indicar la origo era frecuente entre los peregrini, aunque en ocasiones también fue empleada por los ciudadanos romanos, sobre todo con posterioridad al siglo II d. C. ${ }^{73}$. En el caso que nos ocupa el uso de esta fórmula reflejaría bastante bien la situación del mundo tracio, incorporado a Roma de forma tardía y donde la difusión de la vida urbana y la ciudadanía romana se produjeron de forma gradual.

La principal causa de movilidad entre los personajes estudiados fue su servicio en el ejército, dado que la mayoría de los tracios documentados en Hispania fueron militares. Casi todos sirvieron en unidades auxiliares, como sucede con Fuscus Dorilsis (2.1.3), enrolado en una cohors Thracum, y también con Iulius Longinus Doles (2.1.2), que sirvió junto a sus herederos, Fuscus Bitius (2.2.3) y Sulpicius Susulla (2.2.4), en el Ala Tauriana. Por el contrario, hemos identificado únicamente un legionario. Se trata de M. Aurelius Victor (2.1.1), reclutado para la VII Gemina durante el siglo III d. C. Junto a estos militares, que se presentan ante nosotros como el grupo más homogéneo, debemos añadir la existencia de otras casuísticas puntuales, como la del gladiator iSimp(lex)? (2.1.5), llegado a Gades en el contexto de la realización de unos juegos gladiatorios, o la del esclavo Nusatita (2.1.4), documentado junto a su madre (2.2.5) en el municipio de Ossigi. Todos estos casos reflejan una movilidad que no podemos calificar de voluntaria, dado que los militares estuvieron sujetos a las exigencias del servicio y se vieron obligados a desplazarse en función de las directrices emanadas del poder central, mientras que los gladiadores y los esclavos desarrollaron una movilidad que obedecía a los intereses, principalmente económicos, de sus respectivos propietarios y que estuvo condicionada por la existencia de fuertes lazos de dependencia personal.

Para finalizar, realizamos algunas consideraciones sobre la onomástica y el status jurídico de los personajes estudiados. Dejando a un lado los casos de Nusatita (2.1.4) y ¿Simp(lex)? (2.1.5), que presentan nombres sencillos propios del ámbito servil, encontramos en nuestro trabajo una notable variedad onomástica que debemos señalar y de la que podemos extraer tres ideas básicas:

a) Contamos con solo tres casos de onomástica con tria nomina, los de M. Aurelius Victor (2.1.1), C. Valerius Avitus (2.2.2) y M. Aurelius Achaicus (2.2.1). Los dos primeros son ciudadanos romanos, una condición que también podríamos extender a M. Aurelius Achaicus, aunque su cognomen griego permite plantear también la posibilidad de que se trate de un liberto.

b) Son mayoría los individuos con estructuras onomásticas bimembres donde conviven un elemento latino y otro indígena. Así lo observamos en los casos de Fuscus Dorilsis (2.1.3), Fuscus Bitius (2.2.3) y Sulpicius Susulla (2.2.4). Los dos primeros antepusieron el cognomen latino Fuscus a sus posibles nombres indígenas, aunque en el caso de Bitius no es descartable que nos encontremos ante una onomástica invertida. Ambos

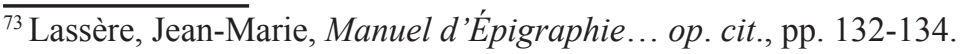


personajes serían probablemente dos peregrini que habrían latinizado parcialmente sus nombres. Por su parte, Sulpicius Susulla porta un gentilicio imperial que podría estar reflejando su acceso a la ciudadanía romana en época de Galba. En este ambiente podríamos situar también a Iulius Longinus Doles (2.1.2), ya que su onomástica, que se compone de tres elementos, presenta un nomen seguido de dos cognomina, uno latino y otro indígena. De su gentilicio se desprende que Doles estaba en posesión de la ciudadanía romana, una circunstancia que contrasta, sin embargo, con su servicio en una unidad auxiliar.

c) Existe una notable diferencia entre los inmigrantes tracios del siglo i y los del siglo III, ya que los primeros —Fuscus Dorilsis, Iulius Longinus Doles, Fuscus Bitius y Sulpicius Susulla - conservan aún en su onomástica ciertos rasgos indígenas, mientras que los del siglo III d. C. - M. Aurelius Victor y M. Aurelius Achaicus - adoptaron una onomástica plenamente romana en la que destaca el empleo del gentilicio Aurelius, que muy posiblemente debamos relacionar con los efectos jurídicos derivados de la promulgación de la Constitutio Antoniniana. 